BMJ Surgery, Interventions, \& Health Technologies

\title{
Outcomes after peripheral artery disease intervention among Medicare-Medicaid dual-eligible patients compared with the general medicare population in the Vascular Quality Initiative registry
}

\author{
Andrea M Austin (1) , ${ }^{1}$ Gouri Chakraborti, ${ }^{2}$ Jesse Columbo, ${ }^{3}$ Niveditta Ramkumar, ${ }^{1}$ \\ Kayla Moore, ${ }^{3}$ Michelle Scheurich, ${ }^{1}$ Phil Goodney ${ }^{3}$
}

To cite: Austin AM,

Chakraborti G, Columbo J, et al. Outcomes after peripheral artery disease intervention among Medicare-Medicaid dual-eligible patients compared with the general medicare population in the Vascular Quality Initiative registry. BMJ Surg Interv Health Technologies 2019;1:e000018. doi:10.1136/ bmjsit-2019-000018

Received 29 July 2019 Revised 21 October 2019 Accepted 29 0ctober 2019

Check for updates

(c) Author(s) (or their employer(s)) 2019. Re-use permitted under CC BY-NC. No commercial re-use. See rights and permissions. Published by BMJ.

${ }^{1}$ The Dartmouth Institute, Dartmouth College, Lebanon, New Hampshire, USA

${ }^{2}$ Analytics Institute, DartmouthHitchcock Medical Center, Lebanon, New Hampshire, USA ${ }^{3}$ Vascular Surgery, DartmouthHitchcock Medical Center, Lebanon, New Hampshire, USA

Correspondence to Dr Andrea M Austin; andrea.m.austin@dartmouth. edu

\section{ABSTRACT}

Objective To determine whether patients from the Vascular Quality Initiative (VQI) registry who are MedicareMedicaid dual-eligible have outcomes after surgical intervention with medical devices such as stents for peripheral artery disease comparable to the outcomes of those eligible for Medicare alone.

Methods The study cohort included fee-for-service Medicare beneficiaries from 2010 to 2015 who underwent peripheral vascular intervention as determined by the VQI. We performed propensity matching between the dualeligible and non-dual-eligible cohorts. Postintervention use, including imaging, amputation and death, was determined using Medicare claims data.

Results Rates of major amputation were higher among dual-eligible patients $(13.0 \%$ vs $10.5 \%, p<0.001)$, while time to amputation by disease severity was similar $(p=0.443)$. For patients with more advanced disease (critical limb ischaemia (CLI) vs claudication), dual-eligible patients have significantly faster times to any amputation and death $(p<0.001)$. For of postoperative imaging, $48.4 \%$ of dual-eligible patients receive at least one postoperative image, while the percentage for non-dual-eligible patients is $47.2 \%(p=0.187)$.

Conclusions Patients with mild forms of peripheral artery disease (PAD), such as claudication, demonstrated similar outcomes regardless of dual-eligibility status. However, those with severe PAD, such as $\mathrm{CLI}$, who were also dualeligible had both inferior overall survival and amputationfree survival. Minimal differences were observed in process-driven aspects of care between dual-eligible and non-dual-eligible patients, including postoperative imaging. These findings indicate that despite receiving similar care, dual-eligible patients with severe PAD have inferior long-term outcomes, suggesting the Medicaid safety net is not timely enough to benefit from long-term outcomes for these patients.

\section{INTRODUCTION}

Nearly nine million people in the USA receive health insurance benefits from both Medicare and Medicaid and are often referred to 'dual-eligibles', given their eligibility for these

\section{Key messages}

What is already known about this subject?

- Medicare-Medicaid dual-eligible patients have worse long-term outcomes following surgical intervention for PAD than Medicare-only patients. These differences are confounded by the severity of disease at the time of intervention.

\section{What are the new findings?}

- Patients with peripheral artery disease (PAD) who undergo surgical treatment at a Vascular Quality Initiative participating centre receive consistent preoperative and postoperative care regardless of Medicare-Medicaid dual-eligibility status.

\section{How might these results affect future}

\section{research or surgical practice?}

- The increased severity of disease for MedicareMedicaid dual-eligible patients at the time of intervention is indicative of delayed treatment. Increasing early access to treatment is key to improving outcomes for these patients.

two sources of social support. ${ }^{1}$ Many dualeligible adults have serious health conditions that require complex medical care, and these conditions often impact their health and healthcare needs more than individuals with Medicare coverage alone. ${ }^{2-4}$ For patients to successfully manage several difficult chronic conditions, they must first be able to access the healthcare system and obtain medical care. Having insurance coverage is an integral first step in this process.

The process to obtain Medicaid insurance is long and complicated. Generally, the process to coverage begins, particularly for those under the age of 65 years, by being approved for supplemental security income (SSI) and then, after a 5-month waiting period, social security disability insurance 
(SSDI) entitlement. Once approved for federal SSDI benefits, beneficiaries are required to wait 24 months from disability entitlement to get Medicare. ${ }^{5}$

One example of a chronic condition that fits into this paradigm is peripheral artery disease (PAD) ${ }^{6-8} \mathrm{PAD}$ affects 8.5 million people in the $\mathrm{USA}^{9}$ and is an important chronic health state that can lead to leg amputation and death if not managed effectively. The association of PAD with poverty is complex. A previous studying looking at carotid artery stenosis among elderly dual-eligible patients found that those with dual-eligible status had higher mortality $(\mathrm{OR}=1.35$ for 1-year mortality for dual-eligible vs Medicare-only) and readmission rates $\left(\mathrm{OR}=1.32\right.$ for 30 -day readmissions). ${ }^{10}$ Lower cost sharing and an expanded scope of covered benefits for beneficiaries dually eligible for Medicaid relative to those with Medicare only could increase healthcare access and limit treatment disparities. ${ }^{11}$ One might hypothesise that Medicare-Medicaid dual eligibility might be associated with improved outcomes for $\mathrm{PAD}$, as having dual insurance coverage might eliminate common hurdles to healthcare access, and lessen observed disparities, including by race, socioeconomic status (SES) and gender, which have been well described in PAD populations. ${ }^{12-15}$ Evidence for this hypothesis is mixed, however, as some studies of patients with dual eligibility suggest access to care and health outcomes were worse comparing dual-eligible patients with those who are non-dual-eligible (Medicare only). ${ }^{16}{ }^{17}$ To better understand the relationship between dual eligibility and outcomes in individuals with $\mathrm{PAD}$, we studied patients who underwent peripheral vascular interventions (PVIs) with medical devices, including stents, within the Vascular Quality Initiative (VQI) registry linked to Medicare claims for long-term outcome analysis. PVIs include, but are not limited to, procedures such as balloon angioplasty, stenting and atherectomy. We hypothesised that the care provided to patients with dual eligibility would be similar-or perhaps even better-than provided to non-dual-eligible patients. To test these hypotheses, we performed analyses to look at outcomes related to the follow-up treatment provided and overall health postintervention in both dual-eligible and non-dual-eligible patients treated in centres that participate in the VQI registry.

\section{METHODS}

\section{Data sources and study population}

We conducted an observational cohort study in which we linked participants in the VQI registry to their Medicare fee-for-service claims data in the years 2010-2015. The VQI is the quality improvement registry for the of the Society for Vascular Surgery and is an Agency for Healthcare Research and Quality-Certified Patient Safety Organisation comprising 18 regional quality improvement groups dedicated to improving the quality, safety, effectiveness and cost of vascular healthcare. The VQI collects, exchanges and analyses data on vascular procedures performed at over 500 participating centres in the USA and Canada. VQI registry data contain granular clinical-level detail captured at the time of the procedure and up to 1-year follow-up. We first identified all patients in the VQI registry who underwent a PVI for PAD ( $n=61547)$ during the years of interest. Next, we linked these patients to the Medicare Master Beneficiary Summary File (MBSF) during the year of their surgery as given by the VQI registry $(n=40490)$. We then identified those who were fee-for-service (FFS), those with full Medicare parts A and B coverage during the year (all 12 months or until death if they died during the year of surgery), with no Medicare advantage during the year of their surgery $(\mathrm{n}=36057)$. We identified those individuals whose surgery was present in the claims within 1 month of the surgery date from the VQI registry from the year 2010 to $2015(\mathrm{n}=27852)$. Finally, we required patients to be fee for service from the year of their surgery onwards during the years $2010-2015$ or until death $(\mathrm{n}=21673)$.

Our main exposure variable was dual-eligible status measured within the MBSF. We considered a patient to be dual-eligible if they were indicated as being fully or partially dual for any month during the year in which they had their surgery. Note that all utilization measures are truncated on 30 September 2015 due to the transition from the International Classification of Diseases, 9th Revision (ICD-9) to the 10th revision for consistency and clarity.

\section{Covariates}

We gathered baseline health characteristics from the VQI registry, including their smoking status (never, past or current) and medication use, including use of ACE inhibitors, aspirin, anticoagulants, beta blockers, statins and platelet p2y12 (p2y) inhibitors. Additionally, from the VQI registry, we obtained the patients' history of comorbid diseases, including diabetes and chronic obstructive pulmonary disease (COPD). We also obtained the clinical indication for surgery. In VQI, indication for surgery is reported separately for each limb. We selected the most severe indication for each patient and then dichotomised into those with mild disease (claudication) versus severe manifestations (critical limb ischaemia (CLI)), omitting patients with no indicated reason.

From the Medicare MBSF, we obtained sociodemographic information, including their age at surgery, gender, race, zip code and Medicare-Medicaid dual-eligible status. We linked the nine-digit zip codes (or five-digit zip codes when nine-digit zip codes were unavailable) to the corresponding 2010 census data to obtain the zip code-level median household income. We also dichotomised patients into being either rural or urban residing based on their zip code. ${ }^{18}$

\section{Outcome measures}

Using the Medicare claims data, we measured postoperative outcomes for the cohort of PVI recipients. The outcomes were measured from the date of discharge for the index surgery, according to the Medicare claims data, over the entire study period or until patient death. Outcomes that occurred during the index hospitalisation were not included. 
Outcomes studied include indicators of any readmission, reintervention, amputation (both minor and major) and postoperative imaging, including computerised axial tomography (CT scan), magnetic resonance angiography, MRI or ultrasound. We also defined categories indicating reason for readmission, including vascular, cardiac and other. We examined overall readmission rates and rates within days of discharge of 30 and 90 days. Additionally, we studied the time to first readmission, the occurrence of a vascular reintervention, amputation and death over the study period. Death was determined from the Medicare MBSF, while the remaining outcomes were determined using Current Procedure Terminology codes in the Medicare Physician/Supplier Carrier file (readmissions, reinterventions, amputations and imaging) and ICD-9 codes in the Medicare Provider Analysis and Review file (readmissions).

\section{Statistical analysis}

Descriptive analysis of differences between those who were dual-eligible during the year of their index procedures (dual-eligibles) versus those who were Medicare eligible only (non-dual-eligibles) were compared using either two-sided t-tests (for continuous variables) or $\chi^{2}$ tests (for categorical variables). Given expected and observed imbalances in factors such as socioeconomics and demographics between the dual-eligibles and nondual-eligibles, propensity score matching was then used to balance observable factors between the study populations. Scores were estimated by using logistic regression to determine the likelihood that a patient was dual-eligible based on the covariates listed earlier.

Propensity score matching was performed using a nearest-neighbour matching algorithm to obtain a $1: 1$ match without replacement between the dual-eligible and non-dual-eligible patients. The match was done using an optimal calliper equal to 0.2 times the $\mathrm{SD}$ of the logit of the propensity scores to match the logit of the propensity scores ${ }^{19}$ Finally, the balance in the observable confounders between the matched cohorts was verified by calculating the standardised difference of means for each factor in the two groups. There is no universally accepted cut-off for standardised differences with $0.1,0.2$ and 0.25 all commonly used to denote an important residual imbalance after matching. ${ }^{20}{ }^{21}$ Cut-offs for determining small, medium and large effect sizes have been proposed as $0.2,0.5$ and 0.8 , respectively. ${ }^{20}$ The relative importance or association of the covariates with the outcomes was also considered when we selected a standardised difference cut-off of greater than or equal to 0.2 to determine balance for the covariates. ${ }^{22} 23$

All analyses were performed using SAS V.9.4 and STATA V.15.

\section{RESULTS}

Characteristics of the cohort undergoing PVI

From 2010-2015, 21673 fee-for-service Medicare beneficiaries underwent PVI within the VQI registry (table 1).
Before propensity matching, 25\% (5414) of patients were dual-eligible in the year of their surgery. Dualeligible patients were less likely to be white $(68 \%$ vs $88 \%$ ), less likely to be male (50\% vs $60 \%$ ) and less likely to be rural residing (30\% vs $37 \%$ ) than non-dual-eligible patients. Additionally, they had more comorbidities, with higher rates of diabetes $(56 \%$ vs $46 \%$ ) and COPD (34\% vs $25 \%)$ and less likely to be prescribed PADrelated drugs, including ACE inhibitors, anticoagulants, aspirin and statins. Finally, they were more likely to be treated with beta blockers and p2y inhibitors (table 1). Given these imbalances, we created a propensity-matched cohort, with 10624 patients remaining in the study, half from each cohort, giving an overall match rate of $98 \%$ $(5312 / 5414)$. A sensitivity analysis was also performed to determine if matching within patient race or stratification by age (over 65 years vs under 65 years) would improve balance, improve the match rate or highlight important differences in outcomes among these groups. Both the match rate and balance between the dual and non-dual populations decreased with the stratified analyses (not presented); thus, we continue with the non-stratified analysis. Finally, we see that at the time of surgery, prior to propensity matching, more dual-eligible patients present with greater severity (CLI, $73 \%$ vs $66 \%$ ) than those who are non-dual-eligible. After propensity matching, we obtained statistical balance in all covariates, including severity at presentation, using a standardised difference cut-off of 0.2 , except race, where we saw a slightly higher percentage of white patients in the dual-eligible cohort $(68.1 \%$ vs $67.6 \%$, standard diff $=0.20$; table 1$)$, a statistical but not clinically meaningful difference.

\section{Amputation-free survival after PVI by dual-eligibility status}

Dual-eligible patients are more likely to have a major amputation $(13.0 \%$ vs $10.5 \%, \mathrm{p}<0.001)$ within the first 5 years after PVI. However, rates of minor amputation, such as toe amputation, between dual-eligible and nondual-eligible patients is relatively balanced in the matched cohorts $(10.0 \%$ vs $8.9 \%, p=0.063)$. We also see the rate of any amputation is higher among dual-eligible patients, largely driven by the differences in major amputations (table 2).

In figures 1 and 2, we see Kaplan-Meier curves for the risk-adjusted matched cohorts for amputation-free survival and overall survival, respectively. Among the PAD-related outcomes, these were the two outcomes in which we did not see balance between dual-eligible and non-dual-eligible patients. We have created separate curves by the severity indicator (claudication vs CLI) to account for observed differences driven by disease severity at time of intervention. In figure 1, dual-eligible and non-dual-eligible patients with mild disease (claudication) have similar curves with respect to time to amputation $(\mathrm{p}=0.442)$. However, when the disease is severe (CLI), we see that dual-eligibles have significantly faster times to any amputation $(\mathrm{p}<0.001)$. Thus, not only are dual-eligible patients undergoing more amputations, 
Table 1 Patient demographics of peripheral vascular intervention recipients by dual-eligibility status before and after propensity matching

\begin{tabular}{|c|c|c|c|c|c|}
\hline & \multicolumn{2}{|c|}{ Prematching cohorts } & \multicolumn{2}{|c|}{ Postmatching cohorts } & \multirow[b]{3}{*}{ Standardised } \\
\hline & \multicolumn{2}{|l|}{ Dual eligible } & \multicolumn{2}{|c|}{ Dual eligible } & \\
\hline & No & Yes & No & Yes & \\
\hline & n (\%) & n (\%) & n (\%) & n (\%) & Differences \\
\hline $\mathrm{N}$ & 16259 & 5414 & 5312 & 5312 & \\
\hline \multicolumn{6}{|l|}{ Age (years) } \\
\hline$<65$ & $1439(8.85)$ & 1869 (34.52) & 1559 (29.35) & $1525(28.71)$ & -0.12 \\
\hline $65-74$ & $6946(42.72)$ & $1794(33.14)$ & $1961(36.92)$ & $1944(36.60)$ & 0.06 \\
\hline $75-84$ & $5564(34.22)$ & $1193(22.04)$ & $1235(23.25)$ & $1269(23.89)$ & 0.07 \\
\hline $85+$ & $2310(14.21)$ & $558(10.31)$ & $557(10.49)$ & $574(10.81)$ & 0.10 \\
\hline Male & $9675(59.51)$ & $2718(50.20)$ & $2783(52.39)$ & $2827(53.22)$ & -0.06 \\
\hline White & 14256 (87.68) & $3668(67.75)$ & $3588(67.55)$ & $3616(68.07)$ & 0.20 \\
\hline \multicolumn{6}{|l|}{ Medication use } \\
\hline ACE inhibitor & $13561(83.41)$ & $4600(84.96)$ & $4527(85.22)$ & $4500(84.71)$ & -0.01 \\
\hline Anticoagulant & 2698 (16.59) & $814(15.04)$ & $785(14.78)$ & $812(15.29)$ & 0.05 \\
\hline Aspirin & $11887(73.11)$ & $3855(71.20)$ & $3729(70.20)$ & $3734(70.29)$ & -0.08 \\
\hline Beta blocker & $9854(60.61)$ & $3355(61.97)$ & $3324(62.58)$ & $3276(61.67)$ & -0.05 \\
\hline Platelet p2y12 inhibitors & $6703(41.23)$ & $2313(42.72)$ & $2175(40.95)$ & $2169(40.83)$ & 0.05 \\
\hline Statin & 11466 (70.52) & 3769 (69.62) & $3612(68.00)$ & 3641 (68.54) & 0.09 \\
\hline COPD & $4101(25.22)$ & $1843(34.04)$ & $1645(30.97)$ & $1629(30.67)$ & 0.07 \\
\hline Diabetes & $7481(46.01)$ & $3013(55.65)$ & $3172(59.71)$ & $3073(57.85)$ & -0.19 \\
\hline Rural & $3726(22.92)$ & $1588(29.33)$ & $1499(28.22)$ & $1460(27.48)$ & 0.08 \\
\hline \multicolumn{6}{|l|}{ Smoking status } \\
\hline Never & $3679(22.63)$ & $1196(22.09)$ & $1407(26.49)$ & $1406(26.47)$ & -0.02 \\
\hline Past & 8695 (53.48) & $1911(35.30)$ & $2038(38.37)$ & 2067 (38.91) & -0.09 \\
\hline Current & 3885 (23.89) & $2307(42.61)$ & $1867(35.15)$ & $1839(34.62)$ & 0.09 \\
\hline CLI & $10652(65.51)$ & $3959(73.13)$ & $3974(74.81)$ & $3983(74.98)$ & \\
\hline Claudication & 5607 (34.49) & $1455(26.87)$ & 1338 (25.19) & 1329 (25.02) & 0.11 \\
\hline $\begin{array}{l}\text { Median household income of } \\
\text { zip code }\end{array}$ & $\$ 58125$ (25138) & $\$ 48398$ (21 034.2) & $\$ 48940$ (18999) & $\$ 49406$ (21236) & -0.16 \\
\hline
\end{tabular}

CLI, critical limb ischaemia; COPD, chronic obstructive pulmonary disease.

but also the negative events are occurring more rapidly for those presenting with more severe PAD at time of surgery compared with non-dual-eligible Medicare beneficiaries. In figure 2, the survival curves show that dualeligible patients die more rapidly postsurgery. However, the difference is only statistically significant among those with CLI (claudication $\mathrm{p}=0.065$, CLI $\mathrm{p}<0.001$ ).

\section{Readmission after PVI by dual-eligibility status}

In both crude and propensity-matched analyses, patients with dual eligibility are more likely to have any readmission $(56.2 \%$ vs $50.8 \%, \mathrm{p}<0.001)$ and to have a readmission within the first 90 days post-discharge $(31.3 \%$ vs $29.0 \%$, $\mathrm{p}=0.010$ ). This effect is smaller, though still statistically significant, for readmissions within 30 days of discharge ( $8.25 \%$ vs $7.17 \%, p=0.011$ ). However, among those with a 30 day readmission, the reason for readmission was balanced between the two groups, notably with vascular complications accounting for $3.88 \%$ and $4.99 \%$ of readmissions among dual and non-dual-eligible, respectively (table 2). For reinterventions, there are similar rates by dual-eligibility status (dual-eligibles $19.5 \%$ and non-dualeligibles $18.4 \%, \mathrm{p}=0.131$ ).

Postoperative imaging surveillance after PVI by dual-eligibility status

For postoperative use related to the care delivered by the providers, including reintervention and imaging, we see non-statistically significant differences in the rates by dual-eligibility status. In terms of postoperative imaging, $48.4 \%$ of dual-eligibles receive at least one postoperative image, while the percentage for non-dual-eligible patients is $47.2 \%(\mathrm{p}=0.187)$. 
Table 2 Postintervention outcomes by dual-eligibility status for the propensity-matched cohorts

\begin{tabular}{|c|c|c|c|}
\hline & \multicolumn{2}{|l|}{ Dual eligible } & \multirow[b]{3}{*}{$P$ value } \\
\hline & No & Yes & \\
\hline & n (\%) & n (\%) & \\
\hline $\mathrm{N}$ & 5312 & 5312 & \\
\hline Any readmission & $2696(50.75)$ & $2983(56.16)$ & $<0.001$ \\
\hline $\begin{array}{l}\text { Readmission within } \\
90 \text { days }\end{array}$ & $1538(28.95)$ & $1660(31.25)$ & 0.0099 \\
\hline $\begin{array}{l}\text { Readmission within } \\
30 \text { days }\end{array}$ & $381(7.17)$ & $438(8.24)$ & 0.0114 \\
\hline \multicolumn{4}{|c|}{ Reason for 30-day readmission } \\
\hline Vascular & $19(4.99)$ & $17(3.88)$ & \\
\hline Cardiac & $30(7.87)$ & $33(7.53)$ & 0.7241 \\
\hline Other & $332(87.14)$ & $388(88.58)$ & \\
\hline $\begin{array}{l}\text { Any major } \\
\text { amputation }\end{array}$ & $555(10.45)$ & $691(13.01)$ & $<0.001$ \\
\hline $\begin{array}{l}\text { Any minor } \\
\text { amputation }\end{array}$ & $474(8.92)$ & $530(9.98)$ & 0.0633 \\
\hline Any amputation & $1026(19.31)$ & $1217(22.91)$ & $<0.001$ \\
\hline Any reintervention & $977(18.39)$ & $1038(19.54)$ & 0.1311 \\
\hline Any imaging & 2505 (47.16) & $2573(48.44)$ & 0.1866 \\
\hline Death & $1216(22.89)$ & $1460(27.48)$ & $<0.001$ \\
\hline
\end{tabular}

\section{DISCUSSION}

In this study, we compared postoperative outcomes between dual-eligible and non-dual-eligible Medicare patients who were treated for PAD within the VQI national registry. We found patients with mild forms of PAD, such as claudication, demonstrated similar outcomes regardless of dual-eligibility status. However, those with more severe PAD, such as CLI, who were also dual-eligible had both inferior overall survival and amputation-free survival. Interestingly, we noted minimal differences in process-driven aspects of care provided to dual-eligible and non-dual-eligible patients, such as the rates of recommended drug therapies, postoperative imaging, surgical reintervention and vascular-related readmissions. These findings indicate that despite receiving similar care (ie, medications and imaging) and risk adjustment using data from a clinical registry, dual-eligible patients with severe PAD have inferior long-term outcomes. This suggests that, although Medicaid coverage may offset additional financial burdens of living with chronic health conditions, additional unmeasured factors may be involved that lead to a higher likelihood of death and amputation.

For patients with PAD, previous studies have suggested an association between poverty, measured in a variety of ways, and severity of presentation at the time of surgery, increased postoperative mortality and major amputation. ${ }^{13} 24$ These differences have been attributed to where patients seek care and adherence to guidelinerecommended follow-up care. The suggestion that disparities in care for patients with PAD are due to differences in how that care is provided is contrary to the trends seen in our study, where we see patients, regardless of dualeligibility status, receiving homogenous preoperative and postoperative care. Additionally, patient-related factors such as race have been attributed to increased risk of PAD and poor outcomes. ${ }^{13}{ }^{25}$ We also performed subgroup analyses including by patient age (65 years and older vs under age 65 years), race and by hospital where surgery occurred. These analyses led us to believe that none of these factors were strong determinants of the outcomes.
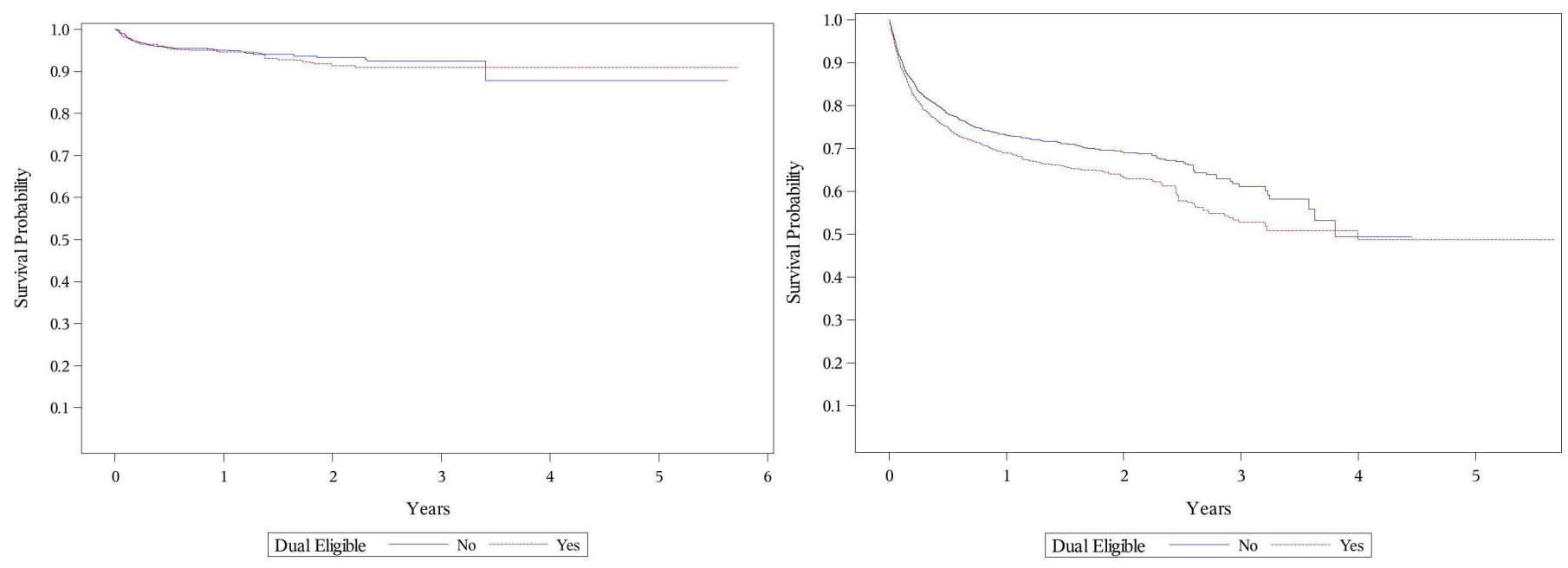

A Claudication

B CLI

Figure 1 Kaplan-Meier amputation-free survival curves by severity, stratified by dual-eligibility status, for the propensitymatched cohorts. CLI, critical limb ischaemia. 


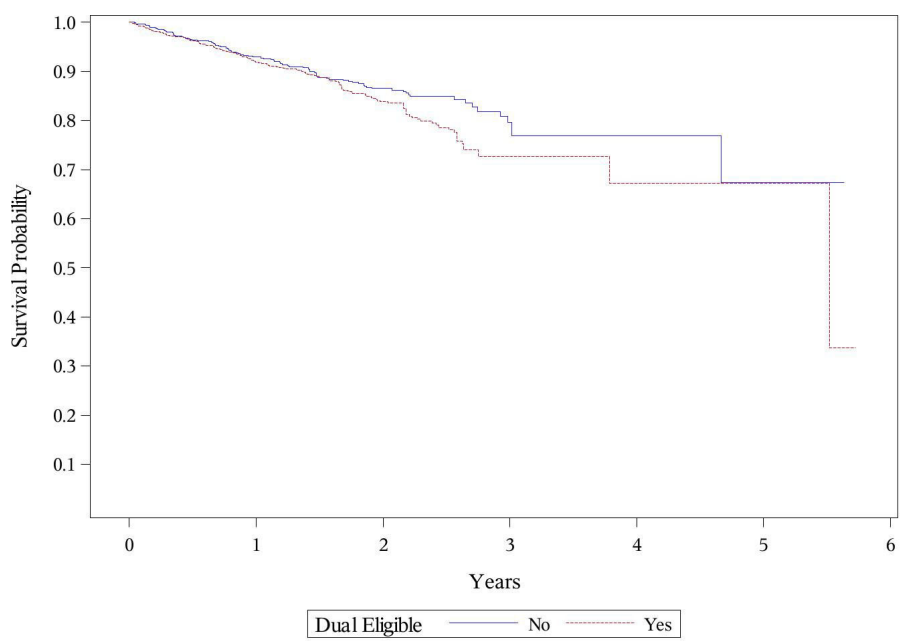

A Claudication

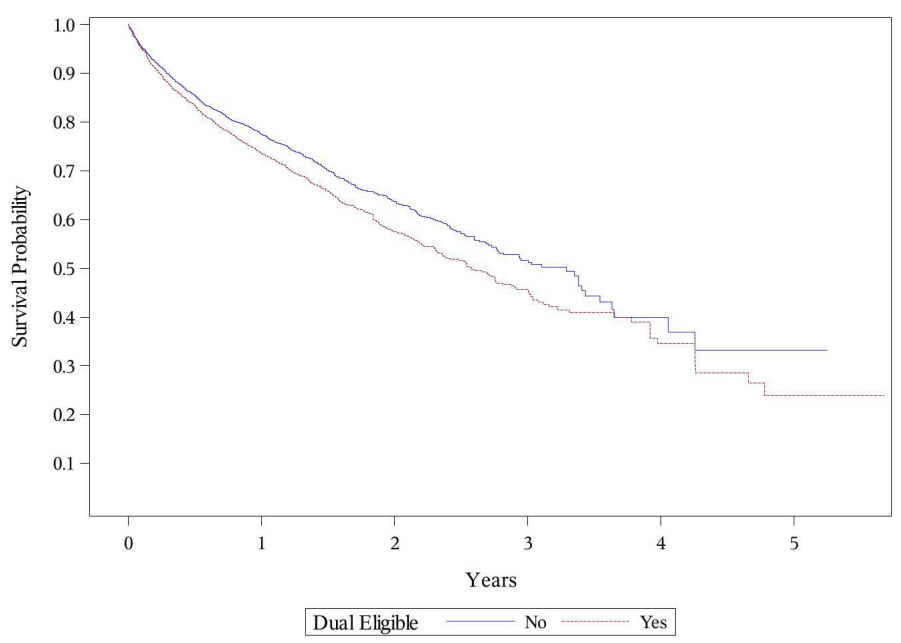

B CLI

Figure 2 Kaplan-Meier survival curves by severity, stratified by dual-eligibility status, for the propensity-matched cohorts. CLI, critical limb ischaemia.

Prior studies have also considered associations between poverty and vascular outcomes. In table 3 , we present a summary of several studies that focused on outcomes for patients with PAD and considered SES as a potential confounder and/or exposure. ${ }^{13}$ 26-28 Table 3 was populated using PubMed searches, including the keywords 'peripheral artery disease', 'cardiovascular disease', 'social determinants', 'socioeconomic status', 'mental health' and 'Medicare-Medicaid dual-eligible'. While table 3 is not exhaustive, it highlights the complex interactions between race, ${ }^{13}$ functional status, ${ }^{27}$ gender $^{28}$ and income ${ }^{25}$ in affecting patient outcomes in PAD. Our study provides context to this prior work by adding more detail to the effect different types of insurance status can have on patient outcomes, even among those with significant comorbid illnesses.

We initially hypothesised that this cohort of patients, regardless of dual-eligible status, would have similar postoperative outcomes. In undergoing invasive treatment for their PAD, the dual-eligible patients with claudicationthe mild form of PAD in this cohort-have several positive influences on their medical care, including insurance, access and quality care. In other words, the 'social safety net' created by Medicare and Medicaid appears to be working. The outcomes surrounding this care, including drug therapies and successful surgeries without reinterventions, followed this hypothesis. However, when we look at time to death and amputation among the patients with CLI-the more severe form of PAD-we see significant differences among those dual-eligible patients. For policymakers, this suggests that in these patients-those who present with the most severe disease-the current social support network may be inadequate. Severity may be confounded by delays in surgery due to waiting times for adequate insurance coverage; this is confounded by the SES of Medicaid patients with known positive correlations between low education and income and health literacy. Low health literacy may contribute to delayed severity at the time of presentation due to patients not pursuing care until their disease is much more advanced due to not understanding their condition well.

The pathway to Medicaid insurance is a long and complicated one for disabled adults and low-income seniors. Medicaid coverage varies across all 50 states. Generally, the process to coverage begins, particularly for those under the age of 65 years, by being approved for SSI, for which only $32 \%$ have their initial application approved, with an average wait time for appeal of 349 days. ${ }^{29}$ Individuals on SSI are eligible for Medicaid but not necessarily Medicare. ${ }^{30}$ There is a 5 -month waiting period to obtain SSDI entitlement, when a person is eligible to start receiving monthly benefits. Once approved for federal SSDI benefits, beneficiaries are required to wait 24 months from disability entitlement (ie, 29 months from the date of disability onset) to get Medicare. ${ }^{5}$ This serious delay in coverage could push a patient with PAD to postpone treatment for financial reasons until coverage is available. Additionally, while these are the programme rules, in practice, due to the lengthy period from application to approval (5 months' minimum and up to several years with appeals), the waiting period does not apply; most people will become eligible for Medicare from the time of eligibility or soon after. The exact pathway to dual eligibility is complex and individualised.

Medicare covers hospital care, outpatient physician care and prescription drugs, while Medicaid reimburses an eligible patient's premiums, deductibles and copays, in addition to paying for nursing home services or community-based support and services like transportation or care at home. Thus, for disabled and low-income individuals, dual eligibility for Medicaid can help to overcome the financial burden of complex treatments for a 


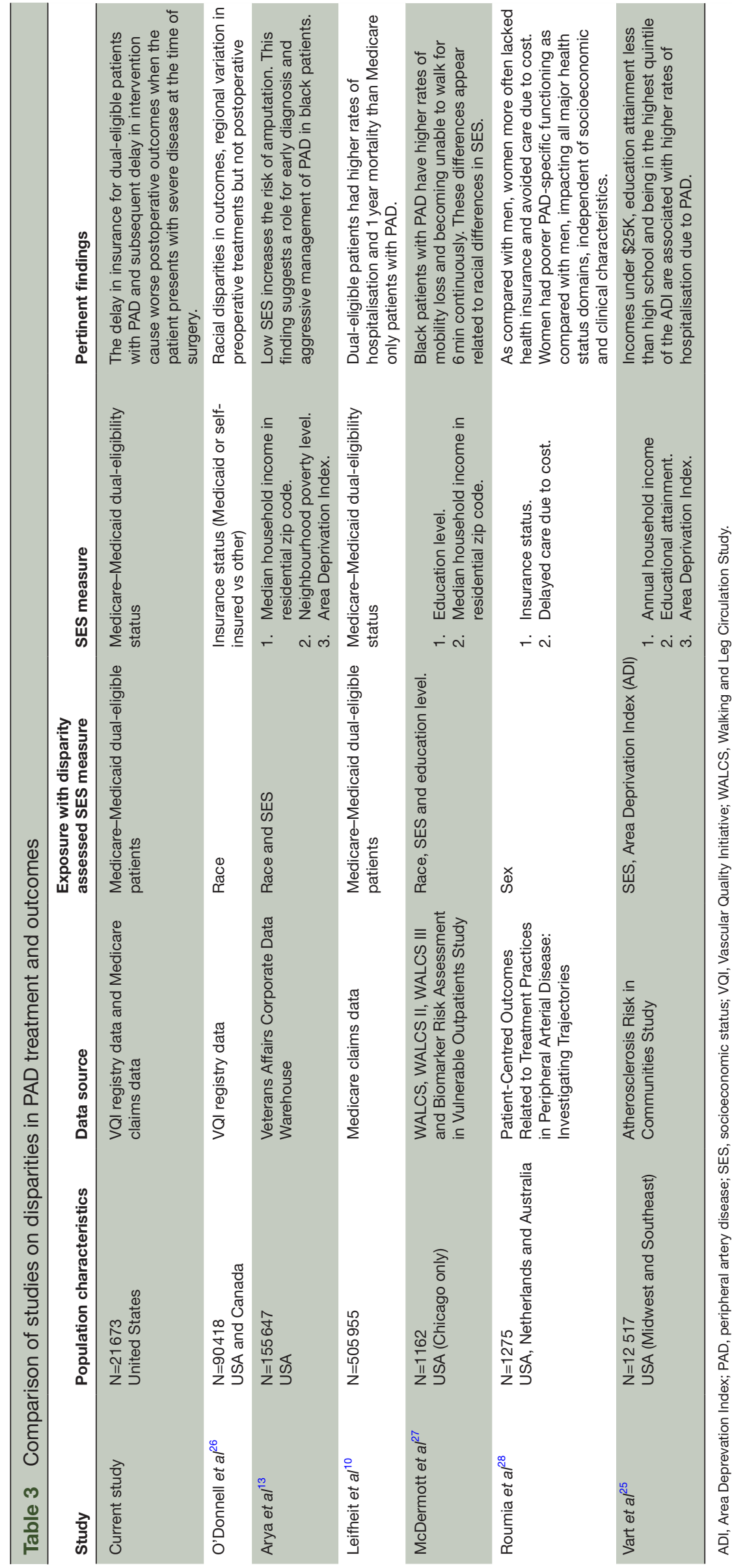


disease such as PAD. Our findings suggest, however, that if patients present with advanced disease, they may not benefit from their insurance, perhaps because the social 'safety net' arrives too late.

There are several important limitations to this study. In our sample, we are using patients who underwent surgery at a VQI-participating hospital; VQI participating centres may have differential outcomes compared with non-VQI hospitals for this particular cohort. This does not necessarily present a balanced picture of what treatment dualeligible undergo across all areas of the USA. Additionally, because we are limited to observing patients starting with the time of surgery, we are unable to unravel what happens to people who (1) do not receive Medicare and/ or Medicaid coverage in time to have treatment, (2) do not undergo treatment at all and the reasons behind this, (3) if there is a delay in care precipitated by a delay in Medicaid and/or Medicare coverage, or (4) if patients received Medicaid prior to their Medicare coverage or vice versa to untangle which insurance precipitated the delay. We have not determined how long the dual-eligible patients have actually been dual-eligible. While we hypothesise the higher severity at time of presentation due to lack of insurance, we have not determined the amount of time the patients have been dual-eligible. While we focused on what happens to patients once they have accessed treatment, understanding how they achieved this is an important next step. Another limitation is the lack of longitudinal data with respect to important factors, such as smoking, and medical control of conditions, such as diabetes, as well as external social and environmental factors, all ultimately influencing outcomes. A final limitation is the inability to assess other forms of supplemental insurance coverage other than Medicaid in the FFS Medicare population that may provide the same financial safety net as Medicaid coverage. Approximately $80 \%$ of Medicare FFS patients have supplemental insurance, approximately $22 \%$ of which is Medicaid. ${ }^{31}$

In summary, better understanding the pathway to and spectrum of care received by patients by dual-eligibility status is an important first step in recognising if the social safety nets provided by Medicaid and Medicare are benefiting those in need to the intended extent. PAD, as an example, is strongly associated with low SES and dual eligibility. Patients appear to undergo similar treatments for PAD whether they are dually eligible or not, and yet outcomes for those with the most severe forms of PAD remain worse for dually eligible individuals when compared with similar patients who are not dually eligible. Future work will focus on clarifying why these vulnerable patients fare worse even despite extensive invasive care for PAD and designing pathways to limit these income and insurance-based disparities.

\section{Twitter Phil Goodney @DartmthSurgHSR}

Contributors AMA, GC, JC, NR, KM, MS and PG contributed to the design, analysis and interpretation of the data and the manuscript content. AMA and PG drafted the manuscript, and all the other authors reviewed the manuscript.
Funding This work was funded with support from the American Heart Association (18SFRN33900147).

Competing interests None declared.

Patient consent for publication Not required.

Ethics approval Our study received institutional review board exemption from the Dartmouth College Center for the Protection of Human Subjects.

Provenance and peer review Not commissioned; externally peer reviewed.

Data availability statement Data may be obtained from a third party and are not publicly available.

Open access This is an open access article distributed in accordance with the Creative Commons Attribution Non Commercial (CC BY-NC 4.0) license, which permits others to distribute, remix, adapt, build upon this work non-commercially, and license their derivative works on different terms, provided the original work is properly cited, appropriate credit is given, any changes made indicated, and the use is non-commercial. See: http://creativecommons.org/licenses/by-nc/4.0/.

ORCID iD

Andrea M Austin http://orcid.org/0000-0002-4404-6065

\section{REFERENCES}

1 KFF. Dual eligible, 2018. Available: https://www.kff.org/tag/dualeligible/ [Accessed 14 May 2018].

2 Congressional Budget Office. Dual-eligible beneficiaries of Medicare and Medicaid: characteristics, health care spending, and evolving policies. Available: https://www.cbo.gov/publication/44308

3 Bennett KJ, Probst JC. Thirty-Day readmission rates among DualEligible beneficiaries. J Rural Health 2016;32:188-95.

4 Coughlin TA, Long SK, Kendall S, et al. Health care access, use, and satisfaction among disabled Medicaid beneficiaries. Health Care Financ Rev 2002 Winter;24:115-36.

5 Laurence B. How long does it take to get Medicare coverage through social security disability (SSD)? Available: https://www. disabilitysecrets.com/dnewsblog/2010/04/how-long-does-it-take-toget-medicare.html [Accessed 14 May 2018].

6 Zaitoun A, Al-Najafi S, Musa T, et al. The association of race with quality of health in peripheral artery disease following peripheral vascular intervention: the Q-PAD study. Vasc Med 2017:22:498-504.

7 Ahmed S, Chowdhury MAH, Khan MA, et al. Access to primary health care for acute vascular events in rural low income settings: a mixed methods study. BMC Health Serv Res 2017;17:47.

8 Ferguson HJM, Nightingale P, Pathak R, et al. The influence of socio-economic deprivation on rates of major lower limb amputation secondary to peripheral arterial disease. European Journal of Vascular and Endovascular Surgery 2010;40:76-80.

9 Centers for Disease Control and Prevention. Peripheral artery disease (pad) fact sheet, 2016. Available: https://www.cdc.gov/dhdsp/data statistics/fact_sheets/fs_pad.htm2018

10 Leifheit EC, Wang Y, Howard G, et al. Outcomes after carotid endarterectomy among elderly dual Medicare-Medicaid-eligible patients. Neurology 2018;91:e1553-8.

11 Medicare eligibility for dual eligible beneficiaries, 2016. Available: https://www.planprescriber.com/medicare-eligibility/medicare-dualeligibility/ [Accessed 14 May 2018].

12 Amaranto DJ, Abbas F, Krantz S, et al. An evaluation of gender and racial disparity in the decision to treat surgically arterial disease. $J$ Vasc Surg 2009:50:1340-7.

13 Arya S, Binney Z, Khakharia A, et al. Race and socioeconomic status independently affect risk of major amputation in peripheral artery disease. J Am Heart Assoc 2018;7. doi:10.1161/JAHA.117.007425. [Epub ahead of print: 12 Jan 2018].

14 Nguyen LL, Henry AJ. Disparities in vascular surgery: is it biology or environment? J Vasc Surg 2010;51:S36-41.

15 Mustapha JA, Fisher BT, Rizzo JA, et al. Explaining racial disparities in amputation rates for the treatment of peripheral artery disease (pad) using decomposition methods. J Racial Ethn Health Disparities 2017;4:784-95.

16 Gimm G, Blodgett E, Zanwar P. Examining access to care for younger vs. older dual-eligible adults living in the community. Disabil Health J 2016;9:431-8.

17 Lubitz J, Pine P. Health care use by Medicare's disabled enrollees. Health Care Financ Rev 1986;7:19-31.

18 Hadden L, Zdeb M. Zip code 411: a Well-Kept SAS secret. SUGI 31 Posters Paper 2005;143. 
19 Austin PC. Optimal caliper widths for propensity-score matching when estimating differences in means and differences in proportions in observational studies. Pharm Stat 2011;10:150-61.

20 Austin PC. Balance diagnostics for comparing the distribution of baseline covariates between treatment groups in propensity-score matched samples. Stat Med 2009;28:3083-107.

21 Harder VS, Stuart EA, Anthony JC. Propensity score techniques and the assessment of measured covariate balance to test causal associations in psychological research. Psychol Methods 2010;15:234-49.

22 Austin PC. The performance of different propensity-score methods for estimating differences in proportions (risk differences or absolute risk reductions) in observational studies. Stat Med 2010;29:2137-48.

23 Austin PC. Goodness-Of-Fit diagnostics for the propensity score model when estimating treatment effects using covariate adjustment with the propensity score. Pharmacoepidemiol Drug Saf 2008;17:1202-17.

24 Ultee KHJ, Bastos Gonçalves F, Hoeks SE, et al. Low socioeconomic status is an independent risk factor for survival after abdominal aortic aneurysm repair and open surgery for peripheral artery disease. Eur $J$ Vasc Endovasc Surg 2015;50:615-22.

25 Vart P, Coresh J, Kwak L, et al. Socioeconomic status and incidence of hospitalization with lower-extremity peripheral artery disease: atherosclerosis risk in Communities study. J Am Heart Assoc 2017;6. doi:10.1161/JAHA.116.004995. [Epub ahead of print: 10 Aug 2017]

26 O'Donnell TFX, Powell C, Deery SE, et al. Regional variation in racial disparities among patients with peripheral artery disease. $J$ Vasc Surg 2018;68:519-26.

27 McDermott MM, Polonsky TS, Kibbe MR, et al. Racial differences in functional decline in peripheral artery disease and associations with socioeconomic status and education. J Vasc Surg 2017:66:826-34

28 Roumia M, Aronow HD, Soukas P, et al. Sex differences in diseasespecific health status measures in patients with symptomatic peripheral artery disease: data from the portrait study. Vasc Med 2017;22:103-9.

29 Does social Secuirty disability deny everyone the first time they apply? 2018 [Accessed 14 May 2018].

30 Does Medicare of Medicaid come with disability? 2018. Available: https://www.disabilitysecrets.com/will-i-get-medicare-medicaid-withdisability.html [Accessed 14 May 2018].

31 Cubanski JDA, Neuman T, Jacobson G. Sources of supplemental coverage among Medicare beneficiaries in 2016, 2016. Available: http://files.kff.org/attachment/Data-Note-Sources-of-SupplementalCoverage-Among-Medicare-Beneficiaries-in-2016 